\title{
Schistosomiasis Control in China
}

\author{
Hong-Chang Yuan
}

\author{
Shanghai Medical University, Shanghai 200032, P. R. of China
}

After three decades' efforts, schistosomiasis japonica were controlled in one-third (4/12) of endemic provinces and 68.2\% (259/380) of endemic counties throughout the country. The remaining 121 endemic counties are located primarily in the lake and mountainous regions. The epidemiological and ecological features of the lake and mountainous areas are different from the other endemic areas.

The major schistosomiasis control efforts in China can be characterized as follows: (1) Application of centralized leadership and management, since schistosomiasis control is a task not only of the Ministry of Public Health, but also of all local governments in the endemic areas; (2) Integration of actions taken by various departments or bureaus, such as agriculture, water conservation and public health; (3) Promotion of mass participation; (4) Organization of strong professional teams; (5) Raising sufficient funds.

Strategies on schistosomiasis control applied in different areas are divided into three levels: (1) In the areas where the schistosomiasis has been successfully controlled, surveillance must be maintained and immediate action should be taken where new infections occur and/or vector snails are found, so that control can be reestablished quickly; (2) In the areas where schistosomiasis has been partially controlled, any residents and/or live-stock infected should be examined and treated promptly with due care, and environment modifying and or mollusciding must be used to eliminate the remaining snails; (3) In the areas where transmission has not been controlled, the main strategv is to control morbidity. Mass or selective chemotherapy with praziquental should be applied to both infected persons and the live-stock, and environment modification for the snailridden areas should be taken but should be coordinated with agriculture where possible. Advance cases must be treated; and epidemics of Katayama fever prevented; water supply and sanitation should be improved and health education emphasized. Annual mass or selective chemotherapy with praziquental both reduces the prevalence rate and decreases the intensity of the infection for inhabitants and live-stock. As a consequence of the therapy a low prevalence rate can be obtained in a short time. The length of such arrangement period can be decided in accordance with the prevalence of the infection before the drug program is begun. Therefore, a maintenance phase is urgently needed. As China's economy expands and people 's living standard rises, schistosomiasis will be controlled more effectively and successfully.

Key words: schistosomiasis japonica - control - China

Schistosomiasis japonica is a parasitic disease which severely harms people's health in China. It used to be endemic in 380 counties of 12 provinces in the south of China. The total snail ridden area was 14.8 billion $\mathrm{m}^{2}$, and twelve million inhabitants were infected with schistosomiasis. Through thirty-years' endeavor since liberation, schistosomiasis has been successful controlled in two provinces, one municipality and one autonomous region and in 158 counties at a local level. Schistosomiasis has also been effectively controlled in 101 counties. These counties made up $68.2 \%$ of total endemic ones. It attracts worldwide attention that schistosomiasis control has made great achievements in China (Wang et al. 1989). At present, the 121 endemic counties are mainly distributed in the lake and mountain regions.

\section{CURRENT GEOGRAPHICAL DISTRIBUTION OF SCHISTOSOMIASIS}

The endemic areas of marshy type is now mainly in Hunan, Hubci, Jiangxi, Anhui and Jiangsu province. The result of nation-wide sampling survey on schistosomiasis in 1989 indicated that the stool positive rate of residents was $14.4 \%$ in Hunan, $12.9 \%$ in Hubei, $5.5 \%$ in Jiangxi and $8.6 \%$ in Anhui. The number of the infected persons in the five provinces in question was estimated at 1.35 million, making up $85.6 \%$ of 1.58 million infected ones estimated for the whole country. The endemic areas of high mountainous subtype are mainly distributed in Yunnan and Sichuan province with the stool positive rate being $16.8 \%$ in Yunnan and $4.4 \%$ in Sichuan. The number of infected population was estimated at 227,000 in the two provinces, accounting for about $14.4 \%$ of the whole popula- 
tion infected in China (Fig. 1). From 1981 to 1989 , the acute cases in the endemic areas of marshy type increased year by year, but there has been a tendency to drop in the past three ycars. In mountainous regions, the incidence seems to have remained steady. The results of the survey in 1991 showed that nation-wide snail-ridden area was 3.63 billion $\mathrm{m}^{2}$, of which there was 3.46 billion $\mathrm{m}^{2}$ in five provinces of lake regions that made up $95.5 \%, 143$ million $\mathrm{m}^{2}$ in two provinces of the mountainous regions, making up $3.9 \%$, and 20 million $\mathrm{m}^{2}$ in water-net regions of plain, making up $0.6 \%$ of the whole nation's snail-ridden area (Fig. 2)

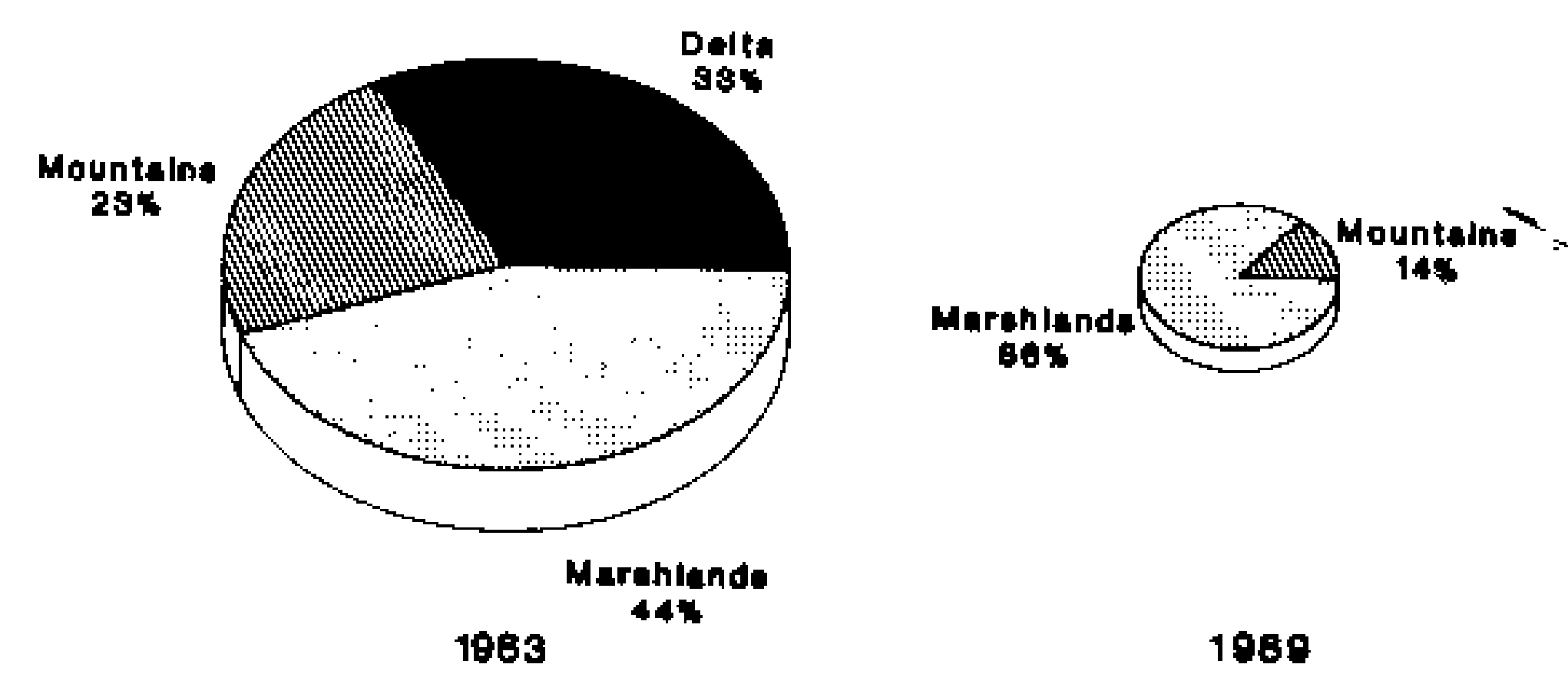

Fig. 1: proportion of infected people with schistosomiasis japonica in different endemic areas of China.

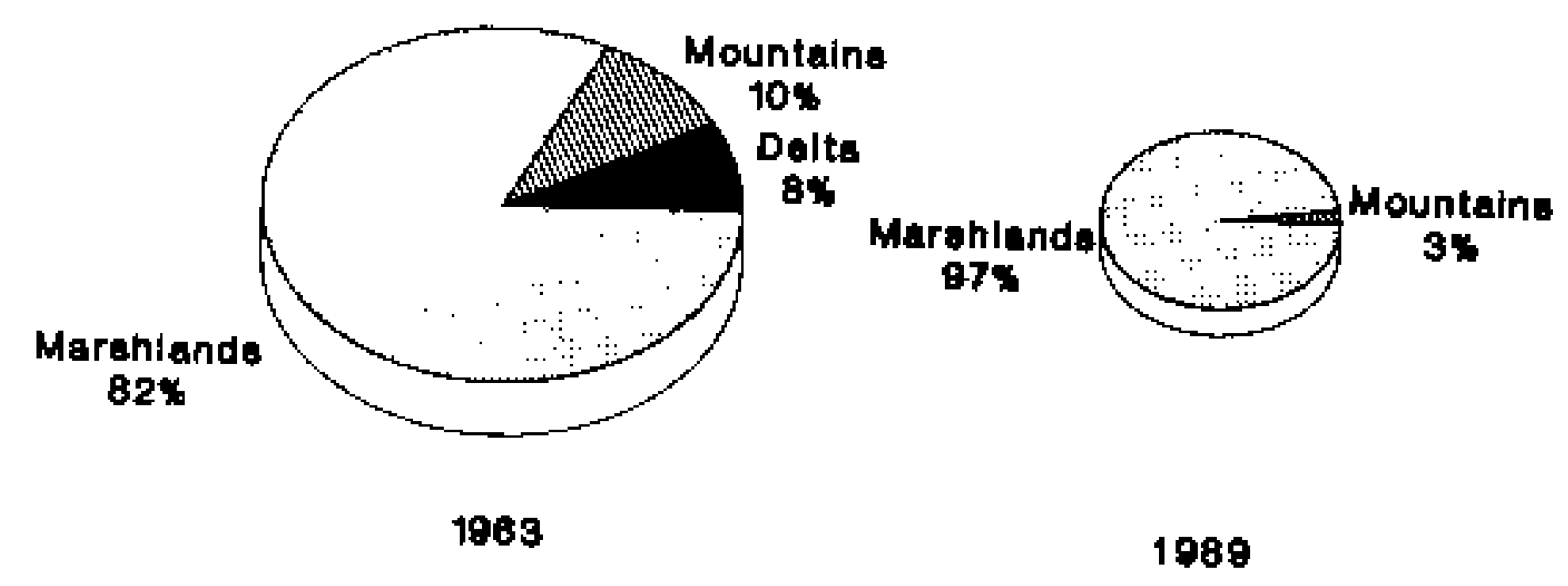

Fig. 2: proportion of snail breeding areas in different endemic regions of China.

\section{EPIDEMIOLOGICAL FEATURES}

The reason that the endemic areas of marshy type and high mountainous subtype become the focal and difficult points of schistosomiasis control is related to the ecological and epidemiological features of schistosomiasis there. Socioeconomic factors have much to do with the transmission of schistosomiasis in these local areas.

There is an apparent seasonal fluctuation of water level appears in the banks alongside Yangtze River and the lakes leading to the River, which dry in winter and flood in summer. The river-lake terrain is overgrown with weeds, and in some areas, large sheet of reeds and willow trees are planted, which is suitable for snail breeding. If they are distributed in irrigation canals inside embankment, the snails' ecological environment is similar to that of the snail in water-net of plain. Thus there are various ways in which human and animals contact with infected water, high frequency of contact, grcat probability of schistosomiasis infection and severity of reinfection. These densely inhabited lake regions are a main base of marketable grain in our country (Yuan et al. 1990, Zhang et al. 1990).

The high mountainous region (the epidemic region of huge mountainous subtype) refers to the high and steep mountainous areas in Yunnan and Sichuan provinces. With mild climate and plentiful rainfall, snails breed in the valley brooks, grassy land of hill slopes and irrigation canals, and on the vertical slop of terraced fields, and so on. Snails are distributed in an independent way, the transmission areas scatter as individual plots. Sometimes, two places are separated only by a peak; one is endemic, and the other is non-endemic. These vast mountainous frontier arcas are thinly inhabited by poor national minorities.

\section{EPIDEMIC FEATURES OF SCHISTOSOMIASIS IN LAKE REGIONS}

Types of endemic area - According to the range of the fluctuation of water level, the sorts of snailridden areas, the distribution of residential quarters and the degree of endemicity, the endemic foci are divided into four subtypes: islet, fork-like lake beach, dam-circle marsh and inner embankment. Schistosomiasis is highly transmissive in islets and some areas of water-net subtype (inside embankment). Before control measures were taken, the infection rate of the inhabitants was $57.9 \%$ and $67.8 \%$ in Nanjishan and Wucheng in Jiangxi Province, $49.7 \%$ in Minglanshan in Hunan Province, $45.3 \%$ and $38.0 \%$ in the villages Guangtang and Zhangjiachi in Hubei Province respectively. In the areas of fork-like lake beach and dam-circle marsh subtypes, the infection rate of population was over $20 \%$ in the residential quarter within $500 \mathrm{~m}$ from the beach. The age distribution of prevalence rate of inhabitants in the islets has two peaks; one is at 10-14 age group and the other is in middle age groups. However, the age distribution of prevalence rate of inhabitants who live near by the lake beach, only has one peak which is at 10-14 age group and keep relatively high level until middle age (Fig. 3).

Exposure and infection - There is a close relationship between the prevalence rate of inhabitants and both the frequency of infected water con- 

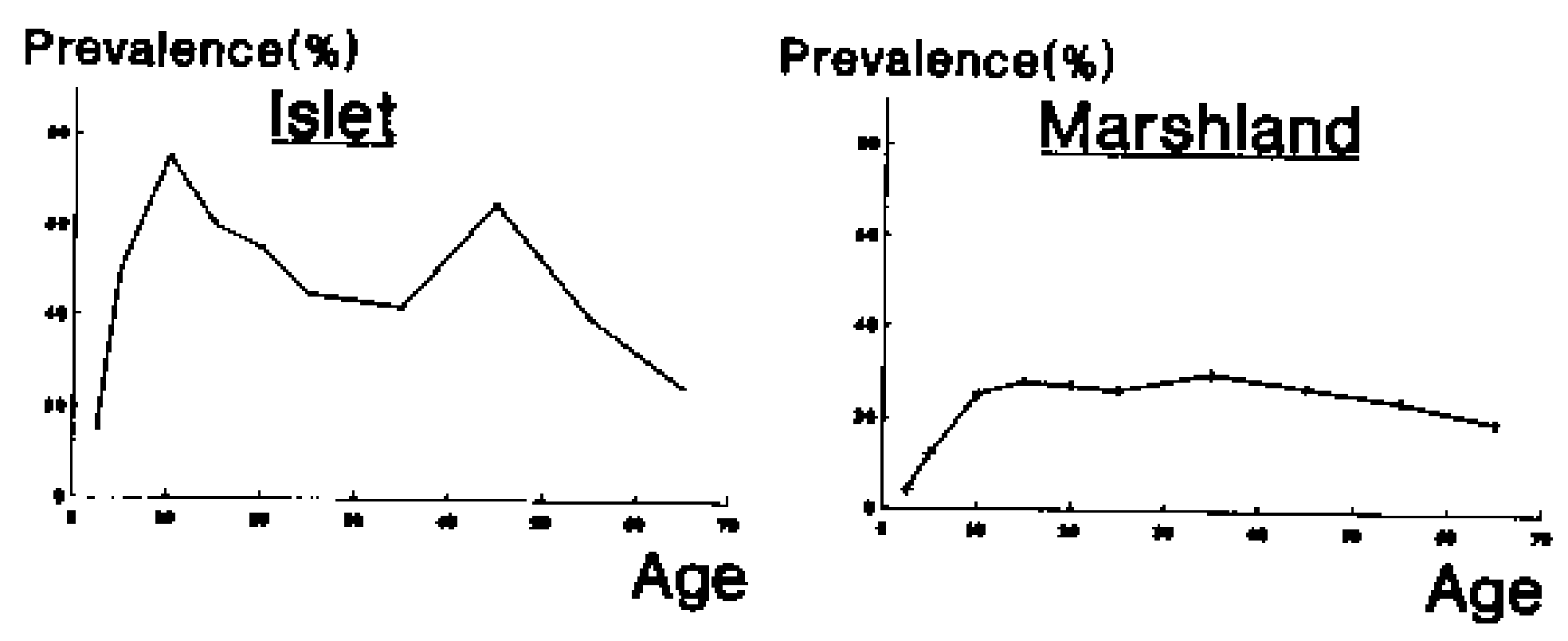

Fig. 3: age distribution of prevalence of schistosomiasis japonica among inhabitants in pilot study areas.

tact and the distribution area and density of infected snails (Yuan et al. 1990, Chen et al. 1990, Wu et al. 1990). It has been investigated that the frequency of infected water contact by the inhabitants on an islet is over 10 times higher than that by those in the areas of marshland outside embankment. Except in February, the frequency is high in each month (Fig. 4). The frequency of contacting with infected water in men is similar to that in women. There is a difference in sex in terms of the frequency of infected water contact in the areas of marshland outside embankment. There, men, generally, contact with infected water more frequently than women. Infected water contact reaches a peak in spring and autumn. Inhabitants near the lake contact with infectious water mainly through domestic and productive activities, whereas the inhabitants far away from the lake contact infected water mainly by productive ones.

High transmissive foci (high risk areas) - It is defined that high transmissive foci are referred to

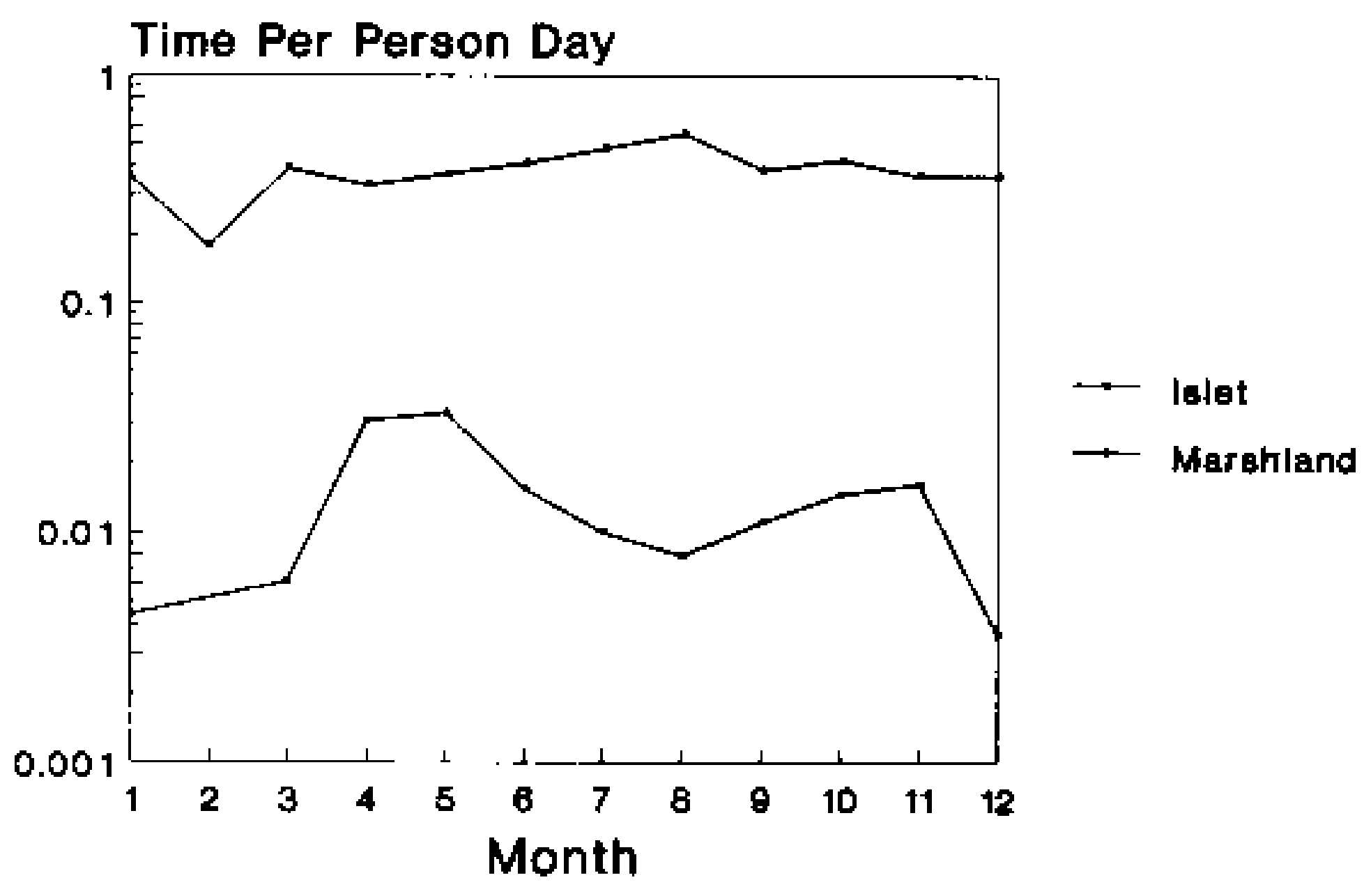

Fig. 4: the frequency of water-contact of inhabitants in pilot study areas. as the places where individuals and livestock often resort to, and where the density of infected snails is high, and also to the water area where schistosome cercariae can be spread. The density of infected snails and the frequency of contact with infected water are two important indexes to define high risk areas. The distribution range of infected snails varies from endemic region to endemic region. The infected snails are concentrated around the villages in the areas of islet and inner embankment water-net subtypes, while distribution of the infected snails has a wider range in those areas of fork-like lake beach and dam-circle marsh subtype, sometimes with a stretch to the beach $1000 \mathrm{~m}$ or greater away. The classification of high risk areas should be made in accordance with the results of a local epidemiological survey.

Main reservoirs of infection - Based on the results of a survey on the lake-beach stools, the indexes of actual contamination by human and livestock and the distribution of infected snails, we have come to a conclusion that there are various kinds of reservoirs in the areas of islet subtype, such as infected pigs, cattle, dogs and residents. Among them the indigenous residents and livestock are the principal reservoirs. The main reservoirs in fork-like lake beach and dam-circle marsh subtype are infected buffalo and cattle or infected buffalo, cattle and pigs, most of the buffalo and cattle coming from other endemic areas. The residents and livestock (mainly buffalo, cattle and pigs) are important reservoirs in the areas of inner embankment water-net subtype.

\section{EPIDEMIC FEATURES OF SCHISTOSOMIASIS IN MOUNTAINOUS REGIONS}

Types of endemic areas - Generally, endemic areas of hill and mountainous types are divided into three subtypes: mountain, hill and basin. After many years of control, schistosomiasis in the endemic areas of hill and basin subtypes has been controlled effectively. At present, in the endemic areas of mountainous subtype, however, there are lot of difficulties in controlling schistosomiasis because of the high mountains, steep slopes, isolated river systems, vast land, sparse population and impassible traffic (Bureau of Endemic Diseases 1990).

Distribution of snails - The snails of mountainous subtypes are characteristic by the distribution along the river systems from upper to low reaches. There is a spotty distribution of snails in upstream, while the distribution range of snails stretches increasingly along the river systems from upper to low streams. The distribution of infected snails is related to the distance to the residential quarters. The snails are distributed most densely in the snail-ridden areas within the distance of 
$100 \mathrm{~m}$ away from these residential quarters. The density of infected snails in the pasturing fields far away from the village is also very high. This probably is due to pasturing activities. The number of cercaria shedding from local snails in mountainous areas is lower than that of snails in waternet areas.

Infection rate of population - In the population of the mountainous areas, there is a high infection rate in the farmers, and children. Infection peak occurs in the age range from 16 to 30 , with the infection rate of men higher than that of women in most regions. But it is reverse in some places, because the frequency of contact with infected water. The infection rate of population in various residential quarters along the same river system tends to increase as the altitude falls in the endemic regions of mountainous subtypes. The intensity of infection is usually lower, the number of advanced patients is smaller and acute cases occur sporadically in this regions.

Principal reservoirs of infection - The livestock outnumbers the residents in the endemic arcas of valley regions of mountainous subtype. Cattle accounts for the majority of livestock. The relative index of transmission (RIT) is $60.9 \%$ for cattle, and $32.8 \%$ for residents. The cattle is the leading reservoirs in the endemic regions, and the residents are the secondary (Zheng et al. 1990). In the basin regions of mountainous subtype, RIT is $87.4 \%$ for human, and $12.3 \%$ for cattle. The residents are the leading reservoirs and the cattle is the secondary one. There are more than ten kinds of wild animals which can acquire natural infection, but this is not an important factor for schistosomiasis transmission. How these animals are capable of preserving the strain of $S$. japonicum needs further studies.

\section{CONTROL STRATEGIES}

The goal of schistosomiasis control in China is to control schistosomiasis in all the amenable areas i.e, to interrupt the transmission.

Through many years' effect, schistosomiasis control in China is now characteristic of unique features.

Centralized leadership and management Schistosomiasis control is not only a task of the Ministry of Public Health, by also a task of all local governments. The state council is in charge of national schistosomiasis control program. The Ministry of Public Health, Agriculture and Water Conservancy join in this task the local government of every province, city and county. A directing group of schistosomiasis control at each administration has been organized with an officer in charge. Schistosomiasis control has been placed in the agenda of each local government and department concerned. There is a professional office where the daily routine is carried out.

Unified program and integrated modification - Schistosomiasis is a highly social disease and schistosomiasis control is a nation-wide systematic project. Executive departments appoint professional staff to deal with the routine. According to duties and tasks, detailed plans have been drawn up. The national and provincial planning committees or departments incorporated schistosomiasis conirol into national and local social developing projects. The agricultural departments take the responsibilities for controlling and treating schistosomiasis of livestock and wiping out snails by uniting this project with agricultural production. The departments of water conservancy treat snail cradication as one of unified projects on the development of water conservancy, drawing up snail eradication programs corresponding to the construction of national and local water conservancy. The departments of public health are responsible for the diagnostic screening and remedy of the disease, the surveillance of endemic situation, the instructions of control technique, and the coordination of the duties carried out by the departments concerned.

Mass participation - Through widespread health education, the residents in endemic areas understand the risks of schistosomiasis, obtain relevant knowledge on prevention and treatment of the disease, cooperate with medical workers for the diagnostic screening and remedy of the disease, and actively join in the activities of schistosomiasis control. The cities and villages in non endemic areas greatly assist in schistosomiasis control with labor, finance and material.

A professional team - The institute of parasitic disease of Chinese Acadcmy of Preventive Medicine, is responsible for professional and technical directions. In each province and city a research institution of schistosomiasis control has been set up in charge of professional routine. In each an endemic county an anti-schistosomiasis station has also been set up. Some of the stations of schistosomiasis control in provinces and counties where schistosomiasis is successful or effectively controlled have joined other stations as a part to continue preventive work and surveillance of schistosomiasis.

Raising required funds - The schistosomiasis control is mainly undertaken by local governments, and the tasks are fulfilled by the state, collective and individual respectively. The funds are raised from the local collection, the world bank loan and the state. During 1991 to $1995,49.3 \%$ from the local authorities, $34.15 \%$ from the world bank loan (counterpart fund was included) and $16.55 \%$ from the state. It takes time to achieve the control of 
schistosomiasis in the whole country in several stages. The period from 1991 to 1995 is the first stage. The aim is to control the endemic situation, decrease greatly the prevalence rate, reduce the epidemic scope, lighten the risk, raise the mass's consciousness of prevention and strengthen institution of schistosomiasis control.

Strategies for control $S$. japonicum was suggested as follows: (1) Surveillance should be strengthened in the areas where schistosomiasis has been successful controlled in case new incidence of the disease and snails are found so that they can be controlled in time; (2) For the areas under effective control, the remaining infected residents and livestock should be examined and treated properly and promptly. And snail-ridden environment should be modified by eradicating the residual snails; (3) In the arcas where transmission has not been controlled, synchronous chemotherapy should be applied to both infected residents and livestock; modification of the snail ridden environment, should be linked with agricultural development and construction of water conservancy; advanced cases, treated; water supply and sanitation, improved and the onset of a large number of acute schistosomiasis. prevented (Mao 1990). When the prevalence rate of inhabitants reduced at a low level after morbidity control, a maintenance phase is needed, otherwise the prevalence rate will re-increase as chemotherapy measure no long used (Jiang et al. 1991).

The "project on the control of schistosomiasis" supported by the world bank loan is an important part of the integrated program of schistosomiasis control in China.

When the intervention measures which include a lot of ethological factors are implemented, sociological factors should be further investigated. At present, the chemotherapeutic strategy applied to human and domestic animals is facing the above situation. It is necessary to enrich and strengthen researches in social, economical, and managerial field which are connected with biomedicine, so as to ensure the quality of intervention measures, and achieve cxpected effects in schistosomiasis control in China.

\section{REFERENCES}

Bureau of Endemic Disease, Ministry of Public Health, P. R. China: 1990. Manual of Schistosomiasis Control. Shanghai Science \& Technology Press.

Chen M, Yuan HC, Jiang QW 1990. Study on the human water contact and socioeconomic factors in endemic area of schistosomiasis japonica. Chin $J$ Schistosomiasis Control 2: 28-33.

Jiang QW, Yuan HC, Zhong DM, Chen $M$ et al. 1991. A longitudinal evaluation of efficacy on control of schistosomiasis by comparative study of different control measures. Chin J Schistosomiasis Control 3: $260-263$

Mao SB 1990. The object, technical measurement and research in control of schistosomiasis. Chin J Schistosomiasis Control 2: 1-5.

Wang HZ, Jia YD, Gou JP, Xia C 1989. Review of schistosomiasis control in last four decades in China. Chin J Schistosomiasis Control 1: 1-4.

Wu ZW, Zhou SJ, Pu KM et al. 1990. The relevance of human behavior to the endemicity and control of schistosomiasis japonica in Dongting Lake. Chin $J$ Schistosomiasis Control 3: 7-11.

Yuan HC, Zhuo SJ, Zhang SJ et al. 1990. Study on the factors affecting the prevalence and epidemiological patterns of Schistosomiasis japonica in the marshlands and lake regions. Chin J Schistosomiasis Control 2: 14-21.

Zhang SJ, Liu ZD, Hu LS et al. 1990. Studies on epidemiological factors and regular pattems of schistosomiasis in Boyang Lake region. Chin J Schistosomiasis Control 2: 17-23.

Zheng J, Qian K, Yao BY et al. 1990. Studies on distribution characteristics of infection sources of schistosomiasis in mountainous regions. Chin $J$ Schistosomiasis Control 2: 24-27. 